\title{
An Investigation of a Sinogram Discontinuity Artifact on Myocardial Perfusion Imaging
}

\author{
Subhash Chand Kheruka ${ }^{1}$, Umesh Chand Naithani ${ }^{2}$, Lalit Mohan Aggarwal ${ }^{3}$, Nirmal Kumar Painuly ${ }^{4}$, \\ Anil Kumar Maurya ${ }^{5}$, and Sanjay Gambhir ${ }^{1}$ \\ ${ }^{I}$ Department of Nuclear Medicine, SGPGIMS, Lucknow, India; ${ }^{2}$ Department of Physics, HNB University, Srinagar, India; \\ ${ }^{3}$ Department of Radiotherapy and Radiation Medicine, IMS, BHU, Varanasi, India; ${ }^{4}$ Department of Radiotherapy, CSMMU, Lucknow, \\ India; and ${ }^{5}$ Department of Radiotherapy, SGPGIMS, Lucknow, India
}

\begin{abstract}
Our purpose was to find out the cause of an unusual distinct break seen on a patient's sinogram despite within-tolerance results on all quality assurance tests during myocardial perfusion imaging (MPI) and corrective measures. Methods: SPECT quality control is a prerequisite to obtaining high-quality diagnostic images. Daily uniformity and energy-peaking tests and weekly center-of-rotation (COR) tests are run to check the performance of the SPECT system. A distinct break in the sinogram of an MPI study was noticed for 1 patient, despite routine quality control tests that showed the system to be well within tolerance limits. Critical inspection of the $\gamma$-camera revealed that the 2 detector heads did not make complete contact with each other at a $90^{\circ}$ position and that a gap of as much as 10.0 $\mathrm{mm}$ was left between the 2 edges of the detectors even though COR testing showed the system to be within tolerance limits. After this gap had been minimized (-10-mm position), the MPI study of this patient was repeated. Results: Reduction of the gap between detectors corrected the sinogram discontinuity. On the MPI study, the break in the sinogram existed because the 2 detectors were not acquiring the data at the same position in their useful fields of view. When one of the detectors was tilted to exactly $45^{\circ}$, the gap was reduced and the data were acquired at the same useful field of view for both detectors. Conclusion: The sinogram artifact may arise even after perfect COR calibration, and in the reported case, the discontinuity in the sinogram was rectified by correcting the angle of the detectors. Meticulous investigation for artifacts must be performed to minimize the probability of false results.
\end{abstract}

Key Words: sinogram; artifact; myocardial perfusion SPECT; COR

J Nucl Med Technol 2012; 40:25-28

DOI: 10.2967/jnmt.111.095562

$\mathbf{M}$ yocardial perfusion SPECT is one of the most common imaging techniques used in nuclear medicine to study cardiac function. Technical and patient-related issues that

Received Jul. 11, 2011; revision accepted Sep. 9, 2011.

For correspondence or reprints contact: Subhash Chand Kheruka, Department of Nuclear Medicine, SGPGIMS, Lucknow 226 014, India.

E-mail: skheruka@yahoo.com

Published online Jan. 25, 2012.

COPYRIGHT @ 2012 by the Society of Nuclear Medicine, Inc. arise during SPECT studies can cause artifacts, which may lead to false scan results that can reduce diagnostic accuracy. Unexpected or transient faults in $\gamma$-camera function may also result in image artifacts. Many of these artifacts are difficult or impossible to recognize without reviewing the sinogram or linogram and may lead to an incorrect interpretation. Therefore, before image processing, raw data usually are reviewed for image quality and patient motion. Image artifacts may appear because of multiple factors, such as patient motion, a shift in the center of rotation (COR), misalignment of the projection frame, differential attenuation in the body, or an unstable photopeak. Before data acquisition, it is also important to visually check the energy window in spectral display mode with the radionuclide to be imaged. In addition, review of the raw images in cine mode may show a flip-flop or flickering pattern dependent on the frequency of the images that have a loss of counts. Review of raw data and sinograms is essential to ascertain the integrity of the SPECT projection data (1).

COR misalignment may also result in image-blurring artifacts in SPECT. Greer et al. reported that the degree of blurring depends on the magnitude of the spatial misalignment of mechanical and electronic COR (2). Therefore, COR calibration is required if distinct discontinuity between the 2 halves of the sinogram is noticed in a myocardial perfusion study. Otherwise, the discontinuity will mimic perfusion defects, an apparent hot spot in the septal region, and flaring from the anterior wall. Regional myocardial perfusion artifacts similar to the problem with COR can also occur if one detector is tilted on a variable-angle multihead camera, because the detector heads will not record exactly the same images (3).

In SPECT, data are acquired over a relatively long time, and during this period the patient might move, resulting in motion artifacts. These motion artifacts can cause quantitative and qualitative image artifacts, which degrade the reconstructed images (4-8). Different methods have been proposed for detection and correction of motion in SPECT studies. One is a motion correction method based on crosscorrelation of linograms or sinograms in SPECT (9). 


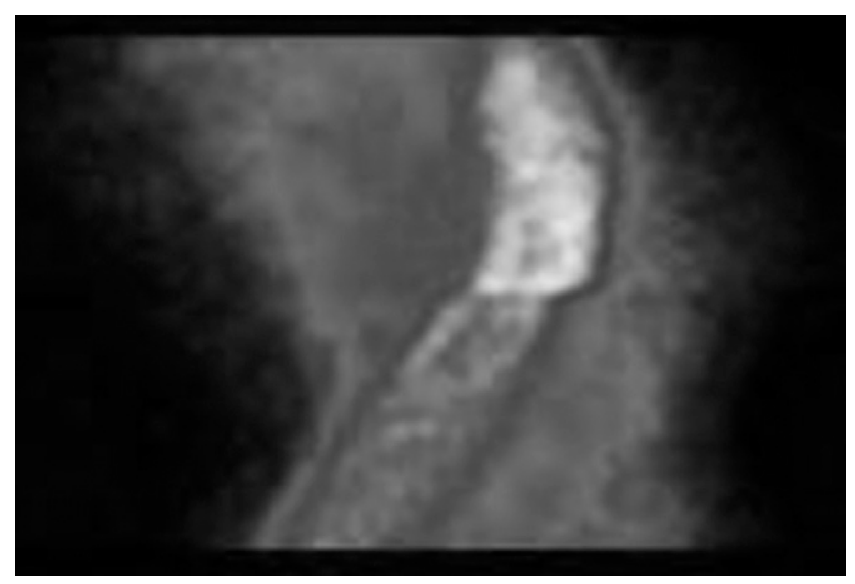

FIGURE 1. Sinogram showing distinct discontinuity.

Artifacts can also be caused by an unstable photopeak position due to spontaneous malfunction of the $\gamma$-camera. This transient photo peak or window shift can be identified on the sinogram image in the final printout of the myocardial perfusion SPECT examination (10).

If there is a timing conflict during data transfer from one computer to another, the computer may produce the discontinuity in the sinogram. A check of the integrity of data transfer is always essential.

To minimize artifacts and obtain high-quality diagnostic images, quality control of the SPECT camera is requisite. Daily uniformity and energy-peaking tests and weekly COR tests must be run to check the performance of system. However, even if all standard tests for various artifacts are run $(11,12)$, there may be discrepancies in the final results because of some unexpected system error. The sinogram or linogram in the final printout can provide important information about COR, patient motion, and off-peak artifacts. Therefore, the sinogram and linogram should be reviewed by the operator to check for motion artifacts (13).
This report highlights a case in which SPECT qualified all the quality control tests but discontinuity in the sinogram was present on myocardial perfusion SPECT. The discontinuity remained inexplicable and persisted in each acquisition.

\section{CASE REPORT}

Myocardial perfusion imaging (MPI) studies were performed as per standard guidelines on a dual-head SPECT/ CT $\gamma$-camera (Hawkeye 4; GE Healthcare) with low-dose CT for attenuation correction. Raw datasets were screened for any patient- or instrument-related artifacts. Only after every possibility of artifacts had been ruled out was further reconstruction of raw images performed (14).

The dual-head SPECT $\gamma$-camera was well calibrated, and the results of all quality control tests were within normal limits.

A distinct break in the sinogram was encountered in all studies (Fig. 1). An area of decreased tracer activity was noted in the apical region, along with a hot spot in the inferior wall (Fig. 2), and wall motion was normal. No improvement in apical activity was noted even after attenuation correction. The same pattern was noted in subsequent patients. Suspicion of some systemic error in the $\gamma$-camera was raised, and routine quality control tests were repeated to rule out calibration and COR errors (15). As all the results were well within tolerance limits, it was clear that this artifact was due to some other, unfamiliar, problem. Critical inspection of the $\gamma$-camera revealed that the 2 detector heads did not come into complete contact with each other at the $90^{\circ}$ position. A significant gap, $10.0 \mathrm{~mm}$, was observed between the edges of the 2 detectors; on minimization of this gap $(-10-\mathrm{mm}$ position), quality control testing, including the COR calibration test, showed the device to be within tolerance limits. The MPI study was repeated on the same patient, and the artifact was found to

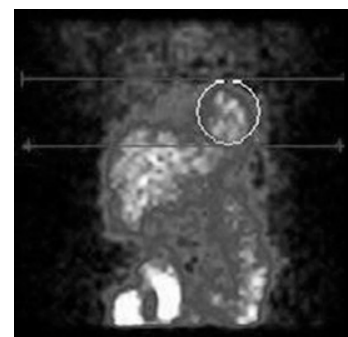

7916,109

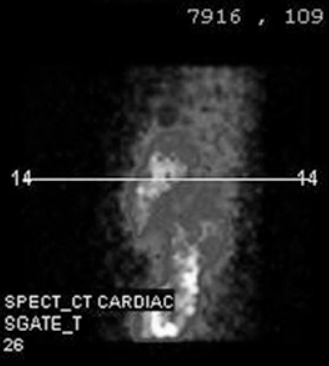

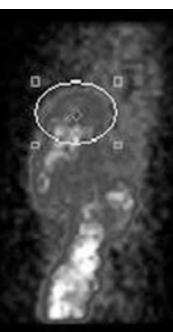

8349,115

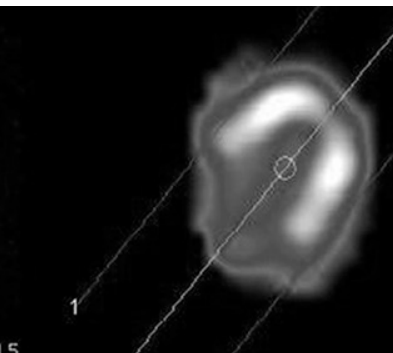

VLA Limits

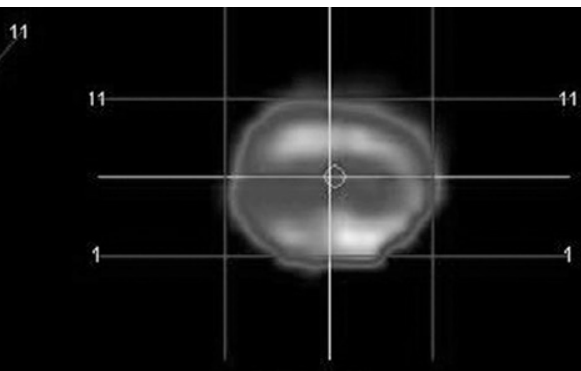

HLA Limits

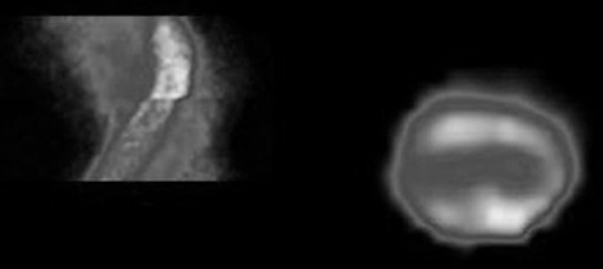

-
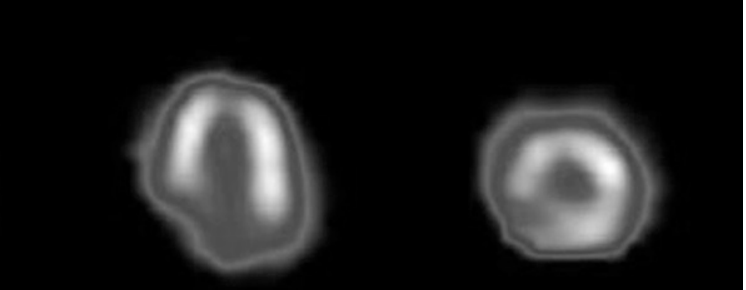

FIGURE 2. Sinogram showing hot spots in inferior region and low count in apical region. 


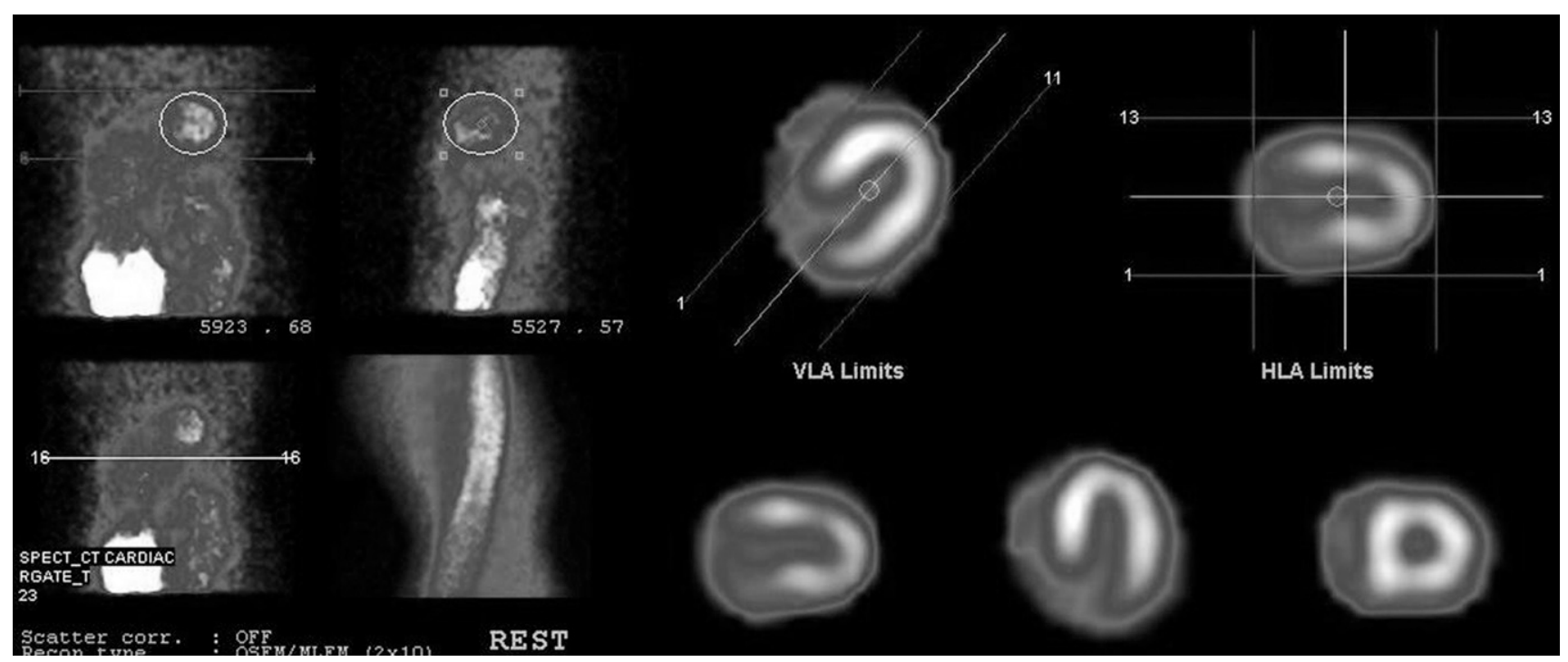

FIGURE 3. Normal sinogram showing uniform horseshoe-shaped myocardium.

have been corrected. The previously noted discontinuity in the sinogram had disappeared; perfusion in the apical region was normal, and the hot spot in the inferior wall had cleared (Fig. 3).

Because the gantry calibration software has a tolerance limit of +10 to $-10 \mathrm{~mm}$ between the 2 detectors, the COR was perfectly within tolerance. For MPI studies, both detectors automatically tilt at $45^{\circ}$ (one clockwise and the other counterclockwise) to make the L shape. In this case, one of the detectors was stopping before completing the $45^{\circ}$ tilt, resulting in a gap between the 2 detectors. Thus, a break in the sinogram was being observed on MPI studies, as the 2 detectors were not acquiring the data at the same position in the useful field of view. When one of the detectors was tilted to exactly $45^{\circ}$, the gap between the detectors was reduced, and data were acquired at the same useful field of view for both detectors.

\section{DISCUSSION}

Myocardial perfusion SPECT is a sensitive tool for the assessment of coronary artery disease. Misinterpretation of a study due to artifacts may lead to a drastic change in patient management. Therefore, it is important to identify errors and minimize false-positive findings. Artifacts may be introduced by errors in equipment, pharmaceutical preparations, reconstruction of images, patient motion, and other factors. A meticulous investigation for artifacts must be performed when a study is being interpreted. It is important for a nuclear medicine specialist to recognize and rectify errors. To do so, the specialist must understand the MPI process and the steps within that process during which errors, artifacts, or pitfalls can be generated and corrected.

To ensure good-quality images, steps must be taken on a regular basis to ensure that the $\gamma$-camera is performing optimally. Some tests need to be performed only monthly or weekly, but other tests, such as uniformity, need to be performed early on the day of the study. Before beginning the patient study, one needs to ensure that the camera has passed all the daily tests and has been corrected for any discrepancy.

Even after adequate calibration of a $\gamma$-camera, artifacts may occur because of patient motion during data acquisition (6), potentially increasing the rate of false-positive findings. Several authors have reported degradation of image quality due to patient motion and violation of COR (16-19).

When nonuniformity is considered to be a possible image artifact caused by a violation of COR, it is recommended that the COR calibration be repeated to resolve the problem. However, in the reported case, the artifact persisted even after the COR had been determined to be perfect. On investigation, the fault could be rectified.

\section{CONCLUSION}

Error identification is essential to minimize false-positive findings. A meticulous investigation for artifacts must be performed during the interpretation of a study. Sinogram artifacts can arise even after perfect results are obtained on quality control tests (COR calibration). In the reported case, the discontinuity in the sinogram was rectified by correction of the angle of the detectors.

\section{ACKNOWLEDGMENT}

No potential conflict of interest relevant to this article was reported.

\section{REFERENCES}

1. Busemann Sokole E. IAEA Quality Control Atlas for Scintillation Camera Systems. Vienna, Austria: International Atomic Energy Agency; 2003:222. 
2. Greer K, Jaszczak R, Harris C, Coleman RE. Quality control in SPECT. J Nucl Med Technol. 1985;13:76-85.

3. Margarita N. Cardiac SPECT. Alasbimn J. 2002;5(18). Article no. AJ18-AJ13.

4. Wheat JM, Currie GM. Incidence and characterization of patient motion in myocardial perfusion SPECT: part 1. J Nucl Med Technol. 2004;32:60-65.

5. Wheat JM, Currie GM. Impact of patient motion on myocardial perfusion SPECT diagnostic integrity: part 2. J Nucl Med Technol. 2004;32:158-163.

6. Tsui BMW, Segars WP, Laush DS. Effect of upward creep and respiratory motion in myocardial SPECT. IEEE Trans Nucl Sci. 2000;47:1192-1195.

7. Chen QS, Defrise M, Deconinck F, Franken PR, Jonckheer M. Detection and correction of patient movements in SPECT imaging. J Nucl Med Technol. 1993;21:198-205.

8. Eisner R, Churchwell A, Noever T, et al. Quantitative analysis of the tomographic thallium-201 myocardial bull's-eye display: critical role of correcting for patient motion. J Nucl Med. 1988;29:91-97.

9. Sarkar S, Oghabian MA, Mohammadi I, Mohammadpour A, Rahmim AA. Linogram/sinogram cross-correlation method for motion correction in planar and SPECT imaging. IEEE Trans Nucl Sci. 2007;54:71-79.

10. Mommennezhad M, Zakavi SR, Sadeghi R, Kakhki VRD. Review of the linogram and sinogram: an easy way to detect off-peak artifacts in myocardial perfusion SPECT. J Nucl Med Technol. 2009;37:188-190.
11. Strauss HW, Miller DD, Wittry MD, et al. Procedure guideline for myocardial perfusion imaging. J Nucl Med. 1998;39(5):918-923.

12. Murphy PH. Acceptance testing and quality control of gamma cameras, including SPECT. J Nucl Med. 1987;28:1221-1227.

13. Lu W, Mackie TR. Tomographic motion detection and correction directly in sinogram space. Phys Med Biol. 2002;47:1267-1284.

14. DePuey EG. How to detect and avoid myocardial perfusion SPECT artifacts. J Nucl Med. 1994;35:699-702.

15. Zanzonico P. Routine quality control of clinical nuclear medicine instrumentation: a brief review. J Nucl Med. 2008;49:1114-1131.

16. Wolf GE. Simulated frame-loss artifacts in myocardial perfusion SPECT imaging: the difference between single- and dual-headed systems. J Nucl Med Technol. 1998;26:248-251.

17. Funahashi M, Shimonagata T, Mihara K, et al. Application of pixel truncation to reduce intensity artifacts in myocardial SPECT imaging with Tc-99m tetrofosmin. J Nucl Cardiol. 2002;9:622-631.

18. Hsu B, Kao CH, Hung GU. Recognition of image artifacts on myocardial perfusion SPECT enhanced by iterative reconstruction. Clin Nucl Med. 2010;35: $445-447$.

19. Bai C, Shao L, Ye J, Durbin M. The effects of center of rotation errors on cardiac SPECT imaging. IEEE Trans Nucl Sci. 2003;50:1528-1535. 